Dominik Ziemke, Kai Nagel, Chandra Bhat

\title{
Integrating CEMDAP and MATSIM to Increase the Transferability of Transport Demand Models
}

Journal article | Accepted manuscript (Postprint)

This version is available at https://doi.org/10.14279/depositonce-7799

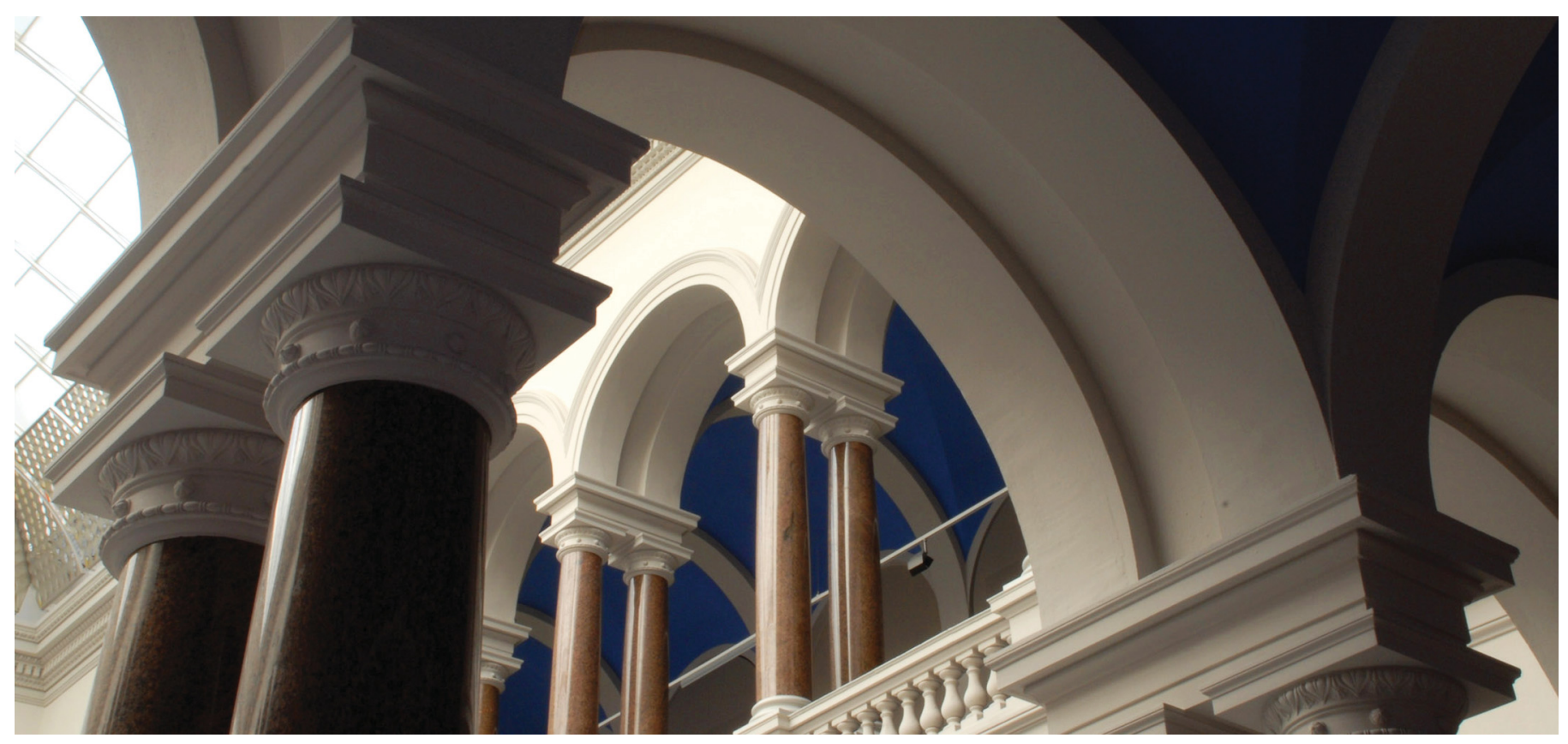

Dominik Ziemke, Kai Nagel, Chandra Bhat, Integrating CEMDAP and MATSIM to Increase the Transferability of Transport Demand Models, Transportation Research Record: Journal of the Transportation Research Board (Vol 2493) pp. 117-125. Copyright (c) [2015] (Sage). DOI: [10.3141/2493-13]. 


\title{
Integrating CEMDAP and MATSIM to Increase the Transferability of Transport Demand Models
}

\author{
Dominik Ziemke, Kai Nagel, and Chandra Bhat
}

\begin{abstract}
An activity-based approach to transport demand modeling is considered the most behaviorally sound procedure to assess the effects of transport policies. This paper investigates whether it is possible to transfer an estimated model for activity generation from elsewhere (the estimation context) and use local area (application context) traffic counts to develop a local area activity-based transport demand representation. Here, the estimation context is the Los Angeles, California, area, and the application context is Berlin. Results in this paper suggest that such a transfer approach is feasible, according to a comparison with a Berlin travel survey. Additional studies need to be undertaken to examine the stability of the results obtained in this paper.
\end{abstract}

Traffic assignment models are useful tools to predict reactions of the transport system to policy measures. Traditional assignment models are static, taking constant origin-destination (O-D) flows as input and producing static congestion patterns as output. To address dynamic policy measures, such as a peak hour toll or changes in the opening times of workplaces or shops, dynamic traffic assignment has emerged as a useful analysis approach (1). Originally, dynamic traffic assignment typically took time-dependent (hourly or day period) O-D matrices as input. More recent approaches [e.g., TRANSIMS (2) or DynusT (3)] often take as input lists of trips in which each trip is defined by the triplet of departure time, departure location, and destination location. It is clear that one can go a step further and take full daily plans as input. To the authors' knowledge, MATSIM is the only model system doing so on a large (regional) scale (4). The advantages of using complete daily activity-travel plans as dynamic traffic assignment inputs include that precedence constraints, such as the fact that a person cannot leave an activity location before having arrived, are automatically resolved. Also, such a model can accommodate more behavioral realism, for example, the willingness to pay for an expensive but faster facility in view of subsequent activity participation.

One question is how the input to such an activity-chain-based traffic assignment model can be obtained. Trip diaries provide the necessary data (i.e., a sequence of departure times, mode choice decisions, and activity locations) directly. A disadvantage of using trip diaries is,

D. Ziemke and K. Nagel, Technische Universität Berlin, Transport Systems Planning and Transport Telematics, Sekr. SG12, Salzufer 17-19, 10587 Berlin, Germany. C. Bhat, Department of Civil, Architectural, and Environmental Engineering, University of Texas at Austin, 1 University Station, C1761, Austin, TX 78712 Corresponding author: D. Ziemke, ziemke@vsp.tu-berlin.de. however, that all information taken from the diaries is by definition not sensitive to policy measures. Also, trip diaries are normally available only for a very small fraction of the population. Another drawback is that in Germany and the United States (and many other parts of the world), the geocoding of the activity location is considered sensitive information under privacy legislation and, thus, increasingly difficult to obtain.

Alternatively, publicly available commuting matrices may be used. However, these matrices do not have a high enough spatial resolution for urban areas. For example, in the publicly available German data, all of the city of Berlin, with 3.4 million inhabitants, is represented by exactly one zone (5). In the United States, commuting matrices are typically available only at a county-to-county level. Since such location aggregation-based matrices may become the rule rather than the exception in privacy-sensitive societies, this factor motivates the search for alternative methods.

So, the question is whether high-resolution O-D information can be generated in some other way. The standard solution would be to estimate an activity location choice model. That approach, however, is difficult if no trip data to estimate the model are available. O-D matrix estimation studies suggest that traffic counts may be used to make an initially rough O-D matrix more appropriate for a region (6). As explained above, however, MATSIM (multiagent transport simulation) is not based on O-D flows but on full daily plans. Thus, the issue becomes whether there could be a source for initial full daily plans for each individual in a region and whether there is a procedure to update these initial full daily plans by using traffic counts. The latter issue may be handled with a procedure proposed by Flötteröd et al. (7) and implemented in the software CADYTS (calibration of dynamic traffic simulations) (8). CADYTS is a procedure to update initial estimates of any arbitrary choice dimension of individual-level travel behavior based on real-world measurements. CADYTS has already been applied to update route choice predictions for car (9) and for public transit (10). However, it has not been used to update full daily activity-travel plans, as is done in this paper. The former issue-a means to generate initial complete daily plans for individuals in a region-is addressed in this paper by using CEMDAP (comprehensive econometric microsimulator for daily activity-travel patterns) (11). In particular, the model parameters of CEMDAP, as estimated for the Los Angeles, California, region (the estimation context), are retained and then used to generate the initial plans for individuals in Berlin (the application context in the current paper). Subsequently, CADYTS is used to update these initial plans with the use of Berlin traffic count data. The main advantage of CEMDAP over other activity-based model (ABM) systems for the generation of the initial plans is that CEMDAP generates full daily activity-travel plans, which is exactly what MATSIM expects as input. Similar attempts with other ABM systems would be 
more difficult as although possibly having daily plans internally, their output consists of hourly O-D matrices (12) or of tours (13). Also, they often do not sample full individuals but rather provide activity chains with fractional weights (13).

In consequence, the objective of this study is to create an activity-plan-based MATSIM transport model for Berlin that is policy sensitive, but at the same time based on freely or easily available data, and uses CEMDAP predictions of initial activity plans combined with Berlin traffic count data. Essentially, an investigation is done on whether it is possible to transfer an estimated model for activity generation from elsewhere (the estimation context) and use local area (application context) traffic counts to develop a local area activity-based transport demand representation.

The issue of transferability has been extensively investigated in the context of trip-based models. A recent review mentions "mixed results regarding the effectiveness and validity of transferability" (14). It also mentions "that transferability improves with a better variable specification and with a disaggregate level model" and that "some level of model updating should be undertaken using local data collected in the application context." ABMs, having an even better behavioral basis than disaggregated trip-based models, should in principle be more spatially transferable than trip-based models. An early study on the transferability of ABM models by Arentze et al. finds good performance for a regionally transferred model in regard to activity participation and time-of-day distributions but weaker results for mode choice (15). As in other studies, model parameters were not updated for the new context (16). Sikder and Pinjari include an updating procedure for the alternative-specific constants in the choice model based on average activity participation rates and average activity durations (17). They find significantly better results with this updating. Bowman et al. combine data from the estimation and application context and test for statistically significant differences to assess whether a variable is transferable (18).

The approach in this study may be viewed as transfer with updating. In contrast to the aforementioned studies, however, the updating operates on initial full daily activity plans rather than on specific model parameters as in traditional transfer updating. In more technical terms, the approach is as follows:

- A synthetic population is generated in the application context, in which each member has the following attributes: age, gender, employment status, being a student, and being retired. For the present study, only people 18 years and older are considered.

- For each working or studying member of the synthetic population, a set of possible workplace and university locations is randomly selected according to the coarse commuting matrix.

- Next, the ABM system CEMDAP generates a full possible daily activity-travel pattern for each possible person-workplace or personschool combination (11). This procedure means that the synthetic persons have multiple activity-travel plans, which are quite different from each other because they all have different work or school locations.

- Finally, the MATSIM transport simulation is run in connection with CADYTS in an iterative loop, in which CADYTS is used to select plans consistent with traffic counts.

This approach is parallel to the O-D matrix estimation. However, instead of entries in the O-D matrix being increased or decreased to match traffic counts, the weights of multiple possible activity-travel plans of each synthetic person are increased or decreased to match traffic counts.

\section{TOOLS}

\section{CEMDAP Model}

For activity-based demand modeling, CEMDAP is used. CEMDAP is a software implementation of a system of random-utility-based models that represent the decision-making behavior of individuals $(11,19)$. Since CEMDAP requires input information on an individual level, which is mostly available only at an aggregate level, synthetic population generation needs to be applied as a preprocess (20). CEMDAP's output consists of the complete daily activitytravel patterns of each individual of the synthetic population and outlines the sequence of activities (and corresponding travel) that a person undertakes during the day $(19,11,21)$. This knowledge is the foundation for transport modeling. As in any market, however, demand is dependent on supply. So, the interaction of supply and demand needs to be modeled.

\section{MATSIM Model}

MATSIM is used to model the interaction of supply and demand on the network by iterating between two major components (4). First, the demand for transport is simulated on the physical network [physical simulation in Figure 1; also referred to as traffic (flow) simulation, mobility simulation (mobsim), or network loading]. Second, the choice processes (decision making) that travelers undertake in reaction to what they experience while traveling are simulated (mental simulation in Figure 1).

Each traveler (agent) makes independent decisions and keeps a record of her or his decisions in a plan that contains the agent's schedule of activities, including times and locations, along with the travel modes.

In the physical simulation, the selected plans of all agents are simultaneously executed. The default physical simulation is a queue model, in which every roadway segment (link) is modeled as a firstin-first-out queue, taking into account the attribute's free-flow speed, link length, flow capacity, storage capacity, and allowed modes (22). An important advantage of the queue simulation is that it can handle sampled populations by scaling down flow and storage capacity accordingly.

In MATSIM, each plan is evaluated according to its performance, which is quantified by a score based on the notion of utility. The utility function encompasses the agents' activity participation and their travel performance (23):

$V(i)=\sum_{\mathrm{ac}, m \in i} V_{\mathrm{act}, m}+\sum_{\mathrm{trav}, n \in i} V_{\text {trav }, n}$

where $V_{\text {act }, m}$ is the utility of activity $m$ and $V_{\text {trav }, n}$ is the utility of travel leg $n$. New scores are calculated only for the selected plan of the current MATSIM iteration. In this study, MATSIM standard scoring parameters are used (23).

Next, the agents decide which plan to execute in the traffic simulation of the next iteration. They may generate a new plan by applying modifications to a copy of one randomly selected plan from their existing plans. Modifications may be done with respect to various choice dimensions (e.g., routing or time choice) through (innovative) strategy modules. If a new plan is created, this plan is marked as the agent's selected plan for the next iteration.

Alternatively, agents may select one of their already existing plans through probabilistic selection and execute it. To do so, a 


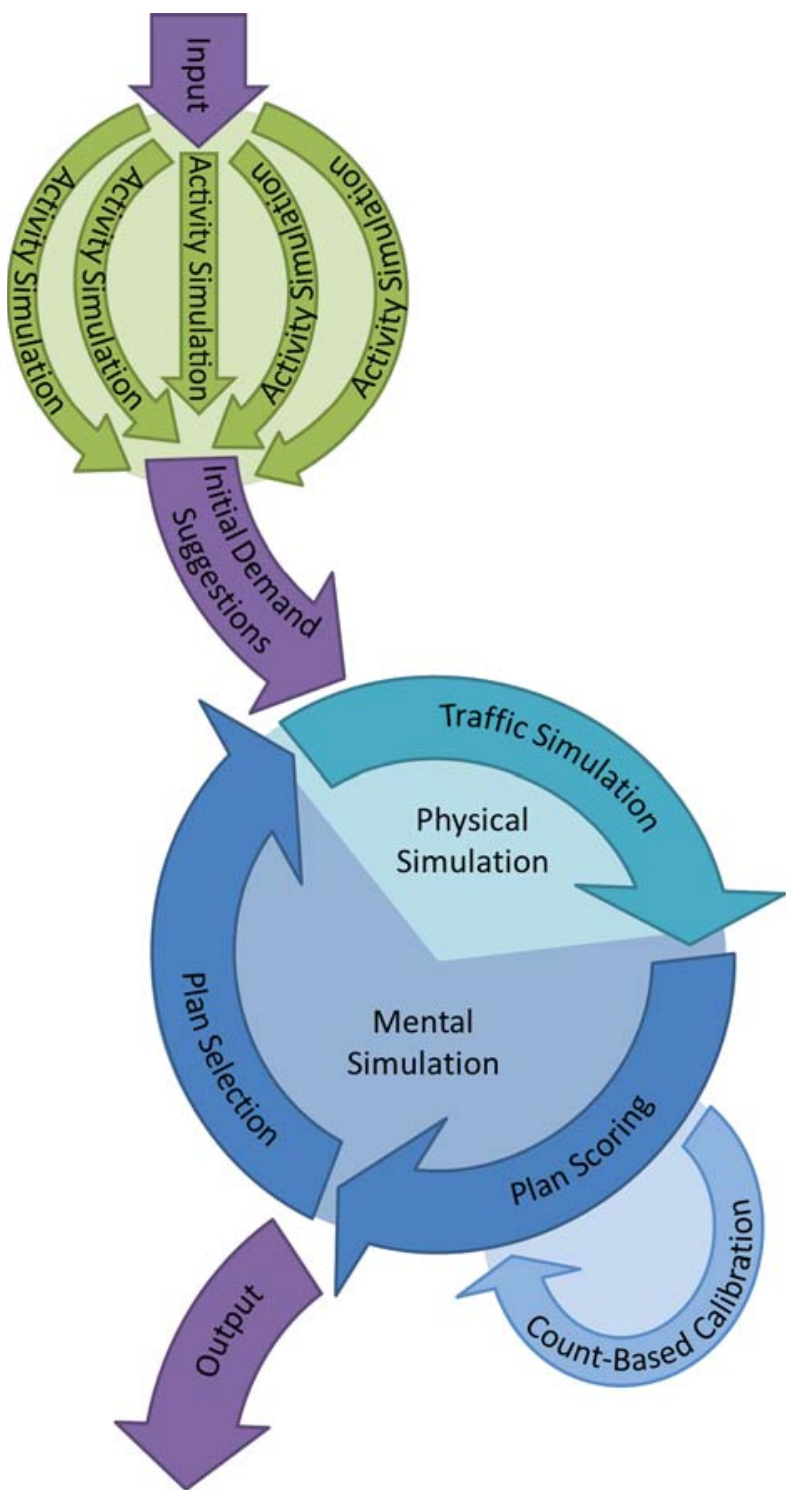

FIGURE 1 Method.

choice from their existing plans is performed by a multinomial logit model, where the selection probability $P(i)$ of a given plan $i$ is related to the plan's score $V(i)$ :

$P(i)=\frac{e^{V(i)}}{\sum_{j} e^{V(j)}}$

where $j$ signifies all plans of the agent under consideration. The iterative optimization process in MATSIM adheres to the concept of evolutionary algorithms. In this approach, transport demand adapts itself to transport supply over the course of iterations.

\section{CADYTS Model}

A drawback of microsimulations is that they-in contrast to analytical models-do not have an explicit mathematical specification, which makes systematic calibration difficult (9). CADYTS overcomes this drawback through its calibration procedure in a Bayesian setting (8). It updates estimates of arbitrary choice dimensions of individuallevel travel behavior according to real-world measurements, for example, traffic counts $(8,9)$.

As stated previously, the probability $P(i)$ of choosing plan $i$ is determined in MATSIM on the basis of the plan scores. Equation 2 can be called the a priori choice probability to choose plan $i$, indicating that this is the plan's choice probability before the measurements are taken into account. To update the plan selection of the synthetic persons, CADYTS combines this a priori choice distribution $P(i)$ with available traffic counts into an a posteriori choice probability $P(i \mid y)(9)$.

As shown by Flötteröd et al., the application of the a posteriori choice distribution requires nothing but adding a plan-specific utility correction to every considered plan of each synthetic person (9). The plan-specific utility corrections are composed of link- and timeadditive correction terms $\Delta V_{a}(k)$. If congestion can be assumed to be light and traffic counts are independently and normally distributed, these link- and time-additive correction terms become (9)

$\Delta V_{a}(k)=\frac{y_{a}(k)-q_{a}(k)}{\sigma_{a}^{2}(k)}$

where

$y_{a}(k)=$ real-world traffic count,

$q_{a}(k)=$ simulated traffic count, and

$\sigma_{a}^{2}(k)=$ variance of traffic count at location $a$ for time bin $k$.

The utility correction of a given agent's activity-travel plan is calculated as the sum of all $\Delta V_{a}(k)$ that are covered by the plan (9). It is calculated for those plans that are selected and simulated in that iteration. With that step, the a posteriori choice probability of plan $i$ of agent $n$ becomes

$$
\begin{aligned}
& P_{n}(i \mid y) \sim e^{V_{n}(i)+\sum_{a k i} \frac{y_{a}(k)-q_{a}(k)}{\sigma_{a}^{2}(k)}} \\
& =P_{n}(i) \cdot e^{\sum_{a k \in i} \frac{y_{a}(k)-q_{a}(k)}{\sigma_{a}^{2}(k)}}
\end{aligned}
$$

where $P_{n}(i)$ is the a priori choice probability of plan $i$ of agent $n$ and $V_{n}(i)$ is the a priori score of plan $i$ of agent $n$ as calculated with Equation 1. Intuitively, if the simulation value, $q_{a}(k)$, is smaller than the measurement from reality, $y_{a}(k)$, an increase in the score and thus an increase in choice probability results. The variance $\sigma_{a}^{2}(k)$ denotes how much one should trust that specific measurement, a large $\sigma_{a}^{2}(k)$ implying a large variance and thus a low trust level. For the present paper, it is assumed that each measurement follows a Poisson distribution [compare Flötteröd (8)], which implies that its expected value equals its variance. This results in

$\sigma_{a}^{2}(k)=\max \left(y_{a}(k), \operatorname{minStddev}{ }^{2}\right)$

where minStddev is a configurable CADYTS parameter, ensuring that the expression does not become too small, which is important for numerical reasons. The utility offset (Equation 3 ) is then embedded as an extra component into the compound MATSIM scoring function (Equation 1) next to activity scoring and travel leg scoring (10). Equation 1 is, thus, modified to

$$
V(i)=\sum_{\mathrm{act} \in m} V_{\mathrm{perf}, m}+\sum_{\mathrm{trav} \in n} V_{\mathrm{trav}, n}+w \cdot \sum_{a k \in i} \Delta V_{a}(k)
$$


where $w$ is the weight of the CADYTS utility correction. By including the adjustments into the score of a plan, the adjustments are memorized for subsequent iterations and stay fixed until the given plan is chosen and, thereby, scored and adjusted again (10).

Conceptually and mathematically, Equation 4 stems from Bayesian statistics, that is, it is a linearized version of the mathematically necessary correction of the behavioral choice probabilities once measurements are available. As one can see, the correction itself behaves as an agent-specific alternative-specific constant (9).

\section{INPUT DATA}

\section{Scenario and Network}

The scenario considered in this study consists of the two German federal states of Berlin and Brandenburg. Transport supply consists of a roadway network, which was created on the basis of data from OpenStreetMap (24). After simplification, the network consists of 11,345 nodes and 24,335 single-direction car-only links.

\section{Synthetic Population}

The synthetic population is based on commuter data provided by the German Federal Employment Agency (5). These data yield the home and workplace municipalities of that part of the working population subject to social insurance contributions. [People subject to social insurance contributions (sozialversicherungspflichtige Beschäftigte) are working people who are not self-employed and whose income exceeds a minimum threshold.]

In this data set, the whole city of Berlin consists of only one municipality, which accommodates 3,375,222 inhabitants (25) and hosts $1,105,037$ socially secured workers (5). Because their home and workplace locations are not specified by the original data in a more detailed way than at the municipality level, Berlin so-called LORs (Lebensweltlich orientierte Räume) (a neighborhood-oriented zone system) are used for the present study. Among other criteria, LORs are spatially defined so that one LOR's population does not fall below or exceed a certain minimum or maximum, respectively (26). Thus, real-world settlement patterns are approximated by selecting LORs randomly for each member of the synthetic population.

Scalings are used to extend the population of socially secured workers to the population of all workers and all nonworking adults. In the current implementation, this population is then scaled by the mode share for automobile since only this mode is considered in the simulation. Each person has the following attributes according to current statistics: employment status, age, gender, being retired, and being a student (27). For analysis, a $1 \%$ sample of this population is used. In future studies, more statistically sophisticated approaches should be used, such as the approach taken by Pendyala et al. (20).

\section{Counts}

For updating the scoring of activity-travel plans, 8,304 hourly count values for 346 count stations are used. Two hundred fifty of these count stations are operated by the Berlin Traffic Management Center (Verkehrsmanagementzentrale), while the remaining 96 stations belong to the motorway administration. In these values, no distinction is made between vehicles of different types (e.g., cars and trucks).

\section{METHOD}

\section{Approach}

As mentioned previously, the idea is to use CEMDAP to generate for each agent a set of several possible daily activity-travel plans, whose parameters have been estimated for another regional context (i.e., the Los Angeles region), and then use CADYTS to select those plans more frequently that are more consistent with measurements from the application context (i.e., the Berlin-Brandenburg region). Several possible daily plans are obtained by running the following two steps multiple times:

1. First, for each member of the synthetic population, a workplace is selected with probabilities according to the commuting matrix. If the workplace falls into the Berlin zone, one of Berlin's LORs is selected randomly. The same is done for school locations (only people 18 years or older are considered).

2. Second, CEMDAP is run with the synthetic population and locations as input as chosen in step 1.

Thus, a set of several possible daily activity-travel plans for each agent is created. As CEMDAP's output is fully disaggregated to the individual-traveler level, it is a perfect match with the requirements of the input plans for MATSIM. Technically, all CEMDAP activitytravel output plans of a given synthetic person are combined into a set of multiple daily plan options of that same person for the MATSIM simulation. For locations, which are specified to the zone level in CEMDAP, coordinates are randomly generated within the zone. From that point, MATSIM's iterative simulation procedure (circular part of Figure 1) is executed as described previously.

\section{Discussion of Method}

Since only automobile traffic is considered in this study, transport mode choice is fixed. Accordingly, the number of motorists needs to be initially correct. Route choice is enabled as a choice dimension with a corresponding strategy module in the MATSIM transport simulation, that is, all agents are able to iteratively create and try out new routes. Location choice and time choice are also seen as fixed from the perspective of the transport simulation, that is, agents cannot create new travel options in relation to timing or location choice during the transport simulation. The special feature of the approach in this study is, however, that agents are still able to adjust their timing or to switch locations among the alternatives they have been provided with by the initial demand suggestions generated by CEMDAP. This feature constitutes a novel compromise between fixed and unfixed choice dimensions. On the one hand, no innovative strategy modules of MATSIM for these choice dimensions are used. On the other hand, the output of CEMDAP can be used as effectively as possible since the decisions concerning these choice dimensions are already conducted by CEMDAP.

Via the mental simulation of the agents' decision making, the demand optimizes itself with respect to supply utilization. CADYTS ties in with the plan scoring process in the mental layer of the MATSIM transport simulation and makes those options prevail that are both reasonable from a behavioral perspective (determined by the activity and leg scoring) and, at the same time, reproduce expected travel patterns (according to real-world measurements). As the influence that CADYTS can exert is obviously dependent on the variety of plans 
each agent possesses, CEMDAP is run multiple times and each output is considered one potential solution. An analogous approach is used by Moyo Oliveros and Nagel, who generate randomized routes of public transport riders as input to MATSIM + CADYTS (10).

\section{RESULTS AND VALIDATION}

More than 100 simulation runs have been undertaken to find the best configuration, which has the following properties:

- Four initial plans seem to be sufficient.

- The maximum number of plans (a MATSIM configuration parameter) should be about twice as high as the number of initial plans.

- Using demand elasticity (i.e., giving each agent an additional initial plan in which the agent stays at home all day) is found to be beneficial to allow the calibration more freedom.

- A flow capacity of 0.02 (i.e., the double of the population scaling value; see discussion) was found reasonable on the basis of indicators such as average (avg.) trip duration (Table 1).

- For the setup of this study, a CADYTS scoring weight of $w=15.0$ should be chosen. Lower values are found not to be influential enough; higher values show first indications of overfitting.

- In contrast to the work of Flötteröd et al., in which CADYTS was applied only for the hours between 6 a.m. and 8 p.m., in the present study CADYTS is applied to all 24 hours of the day (9). Setting the period to 6 a.m. through 8 p.m. showed no discernible differences.

Table 1 depicts the settings and results of the preferred parameter combination of the simulation run, in which the CADYTS updating procedure is applied (column "with CADYTS"). It is compared with an otherwise identical simulation run without updating (column "without CADYTS"). Further, the setting and results of a stability test are shown; a discussion is provided at the end of this section. Finally, reference values from statistics of the study region and a previous study are given (column "reference").

Figure 2 depicts the error graphs of the runs outlined in Table 1. It can be seen that the run with CADYTS updating of plan scoring (Figure $2 b$ ) shows significantly lower mean relative (rel) errors (calculated as the mean relative difference between simulated and measured traffic volumes; depicted in red with squares) with regard to realworld traffic counts. During the daytime, the amount of simulated traffic diverges from the amount of measured traffic on the average by about $20 \%$. Mean absolute (abs) biases (depicted in blue with points) are significantly lower in the case of traffic-count-based updating (note the different scales).

To assess the characteristics of the generated travel patterns, the average values of Table 1 were calculated from the SrV 2008 [System of Representative Travel Surveys (German: System repräsentativer Verkehrsbefragungen)] weekday travel survey for Berlin (28). The values used for validation were calculated directly from the $\mathrm{SrV}$ scientific use files (27). The distribution of trips by time of day and the distributions of trip distances, trip durations, average trip speeds, and activity participation at trip ends are depicted in Figure 3.

Figure $3 a$ shows that the simulation (depicted as a red solid line) has somewhat more traffic during the daytime and a bit less in the evening than the survey (depicted as a blue dash-dot line), which may be explained as follows:

- The midday drop in the survey data neither corresponds to common wisdom from Berlin nor is it contained in traffic counts. Possibly, the survey population behaves differently from the full system. For example, the important demand segment of commercial car traffic is not included in the survey. Presumably, the calibration procedure replaces the missing demand segment by plans that are as close as possible to it.

- The evening drop in the simulation may result from fewer evening activities in Los Angeles compared with Berlin. Presumably, the updating procedure does not have enough suitable plans to converge to observed traffic volumes.

Trip distances (Figure $3 b$ ) are similar, with somewhat more medium-length trips in the survey and slightly more long trips in the simulation. Trip durations behave similarly (Figure $3 c$ ); the steps result from survey participants tending to state "catchy" numbers. Similarly, Figure $3 d$ shows that speeds are similar, with somewhat more medium-speed trips in the survey. The distribution of activities at trip ends is met quite well (Figure 3e). Notably, there is no specific mechanism in the simulation-calibration process that caters for the correct shares of activity types.

To investigate the result as a starting point for policy analysis, a stability test was done. Only the plans from the final iteration were retained, their routes were removed, and the system was then iterated again toward steady state convergence-without looking at the counts anymore, but with route choice enabled. The result is shown

TABLE 1 Settings and Results of Simulation Without and With CADYTS, Stability Test, and Reference Values

\begin{tabular}{lcccc}
\hline Parameter & Without CADYTS & With CADYTS & Stability Test & Reference \\
\hline Demand elasticity & Yes & Yes & Yes & na \\
Number of plans & 10 & 10 & 10 & na \\
Number of initial plans & 4 & 4 & 1 & na \\
Flow capacity factor & 0.02 & 0.02 & 0.02 & na \\
CADYTS scoring weight & 0 & 15 & 0 & na \\
Calibration time & natima & na & na \\
Mean weighted squared error (7) & 219 & 23.00 & 54 & $20(7)$ \\
Car trips (million) & 3.98 & 2.92 & 3.9 & $3.2(26)$ \\
Car trips/person & 3.9 & 11.0 & 11.0 & $3.4(26)$ \\
Average trip distance (km) & 12.0 & 22.0 & 20.9 & $9.5(26)$ \\
Average trip duration $(\mathrm{min})$ & 27.0 & & & $22.3(26)$ \\
\hline
\end{tabular}

NoTE: na $=$ not applicable. 


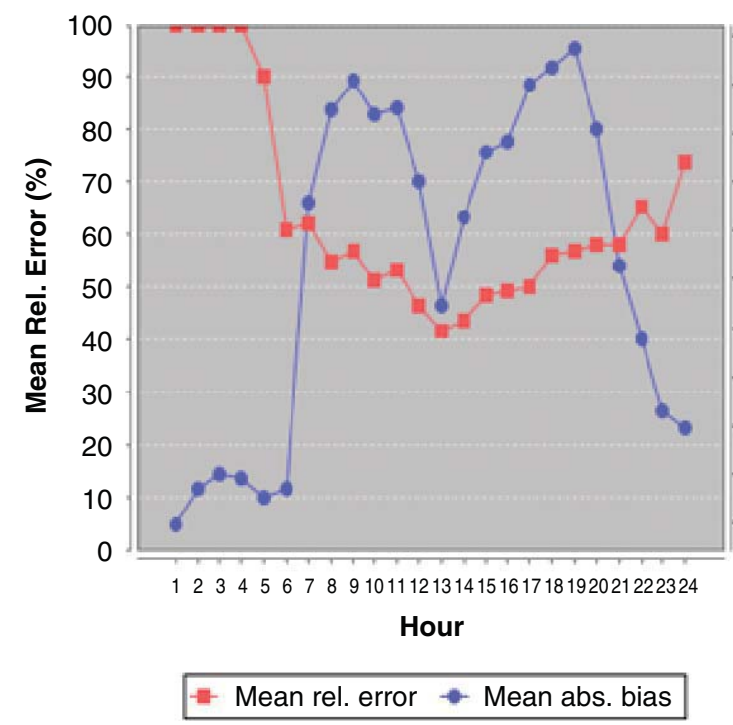

(a)

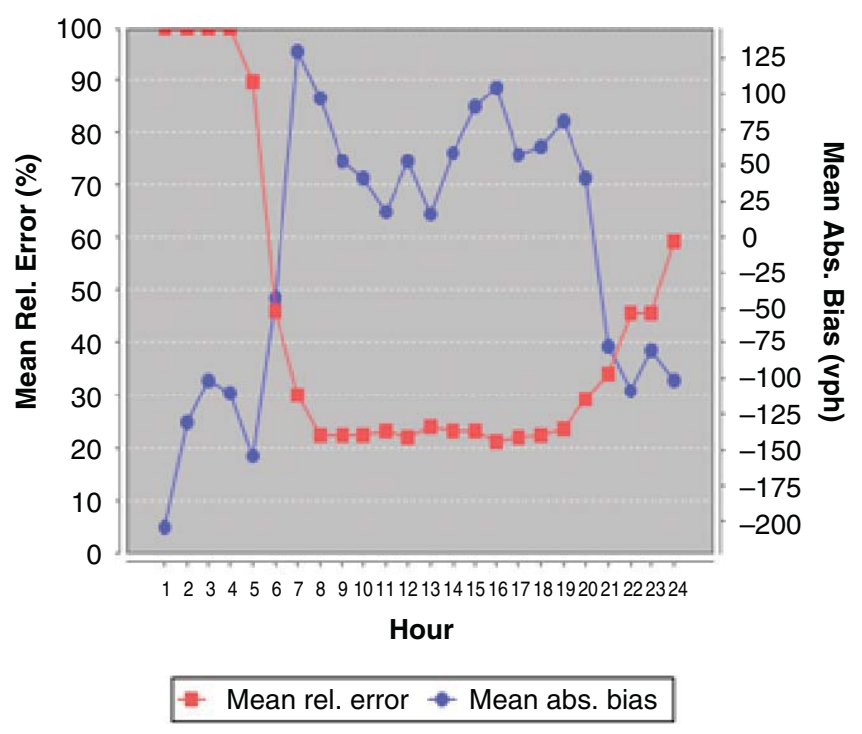

(b)

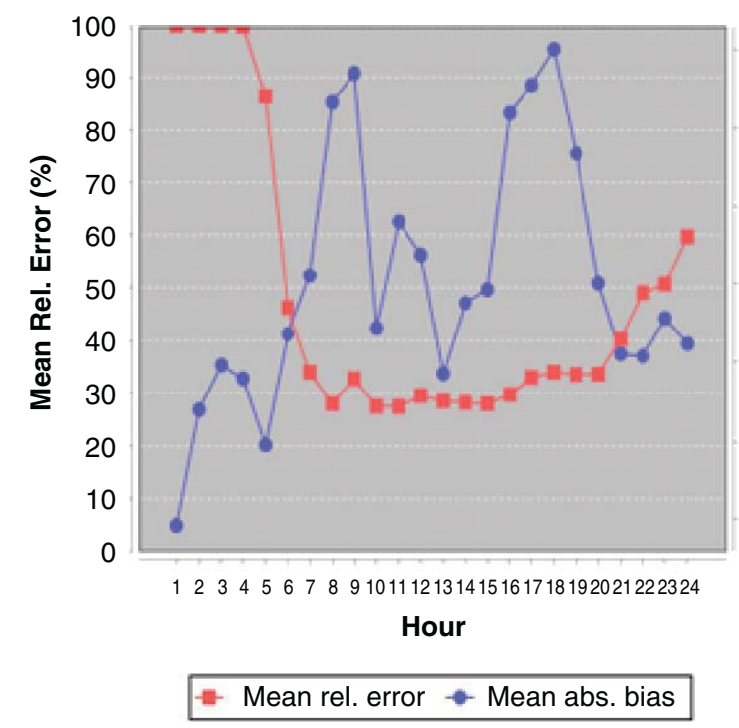

100

50

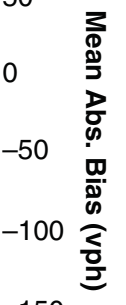

$-150$

$-200$

(c)

FIGURE 2 Error graphs comparing simulated with measured traffic counts: $(a)$ simulation without CADYTS, $[b]$ simulation with CADYTS, and $(c)$ stability test.

in Figure $2 c$ and Figure 3 (in orange). Clearly, departure times (Figure $3 a$ ), beeline distances (Figure $3 b$ ), and activity types (Figure $3 e$ ) cannot change between simulation and stability test. Accordingly, there are also no changes in the results. However, the traffic flow patterns (Figure $2 c$, Figure $3 c$, and Figure $3 d$ ) change only marginally. The indication is that the activity chains that result from the combined CEMDAP + MATSIM + CADYTS application result in stable traffic patterns even when CADYTS is now switched off and route choice is enabled. This step is a first and very important one toward the creation of plausible activity chains for an application scenario accomplished without having used travel diary data from the application context itself. The issue of policy sensitivity is discussed further in the next subsection.

\section{DISCUSSION}

\section{Policy Sensitivity}

As explained in the previous section, the outcome of the process is stable when the behavioral dimension of route choice is opened up. That is, policy measures in which the user reaction can be expected not to go beyond route choice can already be investigated.

For additional choice dimensions, one can calibrate the MATSIM scoring function such that the obtained simulation outcome remains stable when these choice dimensions are opened up and traffic measurement input is not considered any longer. For mode choice and for (departure) time choice, this calibration can be done manually 


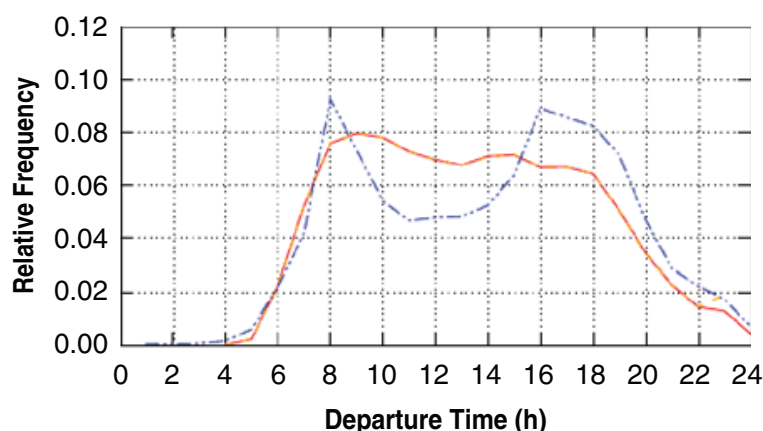

Simulation Stability test

(a)

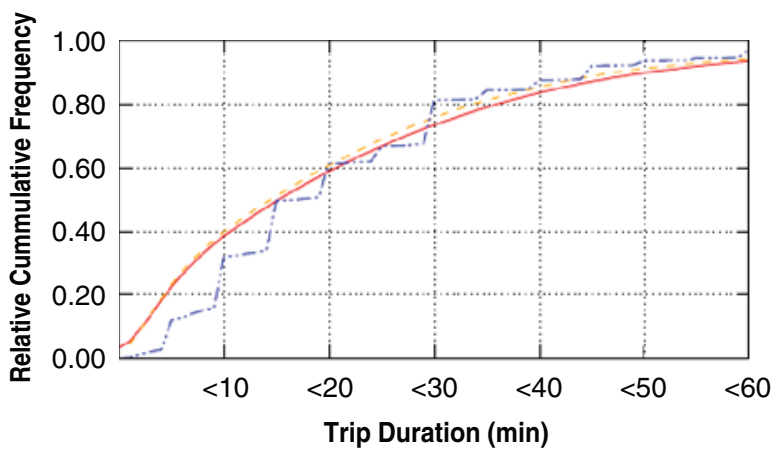

Simulation Stability test Survey $-\cdots-\cdots$

(c)

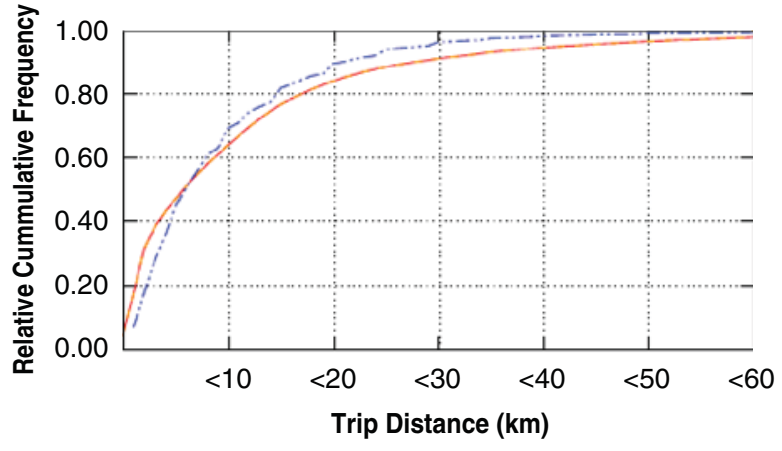

Simulation Stability test

Survey -..-...

(b)

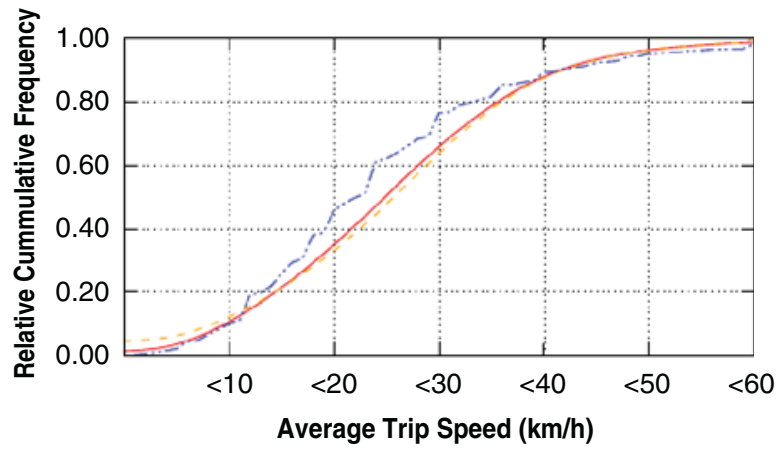

Simulation Stability test

Survey -......

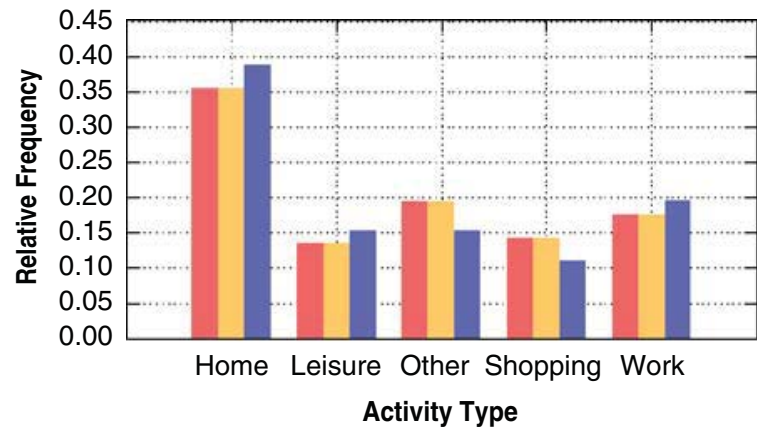

Simulation Survey

Stability test

(e)

FIGURE 3 Comparison of simulation (with CADYTS updating), stability test, and survey: $(a)$ departure times, $(b)$ trip distances, $(c)$ trip durations, $(d)$ average trip speeds, and $(e)$ activity types at trip ends.

with reasonable success [compare Neumann et al. for mode choice (24)]. It is a goal of future research to do this calibration automatically; for an early version see Flötteröd et al. (29). Note that a MATSIM model starting from trip diaries faces the same issue: having trip chains does not mean that the model is policy sensitive. To include mode choice, first a full population rather than a car-only population needs to be created to maintain conceptual soundness.

\section{Activity Pattern and Location Choice}

An interpretation of the current approach is that it first performs coarse location choice and activity pattern generation based on the coarse commuting matrix and on CEMDAP output and then fine-tunes the initial location choice (and possibly the activity patterns) on the basis of the traffic counts, and from then on keeps the 
locations fixed. Clearly, one can consider further modeling options in the upstream models, including the following:

- Rerun CEMDAP on the basis of congested travel times or possibly iterate between MATSIM and CEMDAP.

- Use a better workplace location choice model.

- Include destination side supply constraints.

These issues can be addressed while transferability is maintained, that is, without having to resort to scenario-specific approaches. Some preliminary, more detailed comparisons between simulation results described above and the Berlin survey indicate that some aspects, such as activity participation as a function of age, transfer well, while others, such as participation in the workforce around retirement age, show weaker performance.

\section{Flow Capacity Factor}

The flow capacity of the traffic system was overestimated by a factor of two ( 0.02 compared with a $1 \%$ population). This was done to obtain plausible average trip times-22 rather than 78 min with a flow capacity factor of 0.01 . A preliminary attempt with a $10 \%$ population and a capacity flow factor of 0.1 resulted in a much reduced average trip time of $47 \mathrm{~min}$; there will probably be further improvement with a full $100 \%$ sample. The reason is arguably that the current version of the MATSIM queue model, when run with a reduced flow capacity, generates plausible traffic jam patterns but overlong travel times. This effect needs to be investigated in more detail.

\section{Heavy Goods Vehicles}

The urban Berlin counting stations differentiate between cars and trucks, while those on the motorways do not. For the present study, the car and truck counts for the urban values were added together. In future studies, truck traffic will be considered separately. The CADYTS software allows for doing so. It will just register separate synthetic measurement devices for trucks and cars in cases in which these exist in reality and consider the effect of each plan on these measurements. Essentially, except for indirect congestion effects, plans for cars will not affect measurements for trucks and vice versa. Clearly, some model will have to be devised for truck traffic.

\section{CONCLUSION}

The commuting matrix, either as input to the generation of an O-D matrix or as input to the generation of an activity-based demand, is often not available or not available with high enough spatial resolution. So, destination choice models are often used, which are, however, associated with problems such as lack of suitable input data. In both cases (with or without a destination choice model), it is common to use traffic counts to further calibrate the O-D matrices.

When assignment models are not driven by O-D matrices but by synthetic individual travelers with individual plans, the O-D estimation technique is not directly usable. It is, however, possible to generate multiple plans per person, each having different activity locations, and then to use a Bayesian correction scheme to influence the plan choice probabilities toward measurement data. The procedure was developed and implemented by Flötteröd $(7,8)$ but has so far been applied only to route choice, for car (9) and for public transit (10). In this paper, it is now applied for the first time to activity plan choice, which includes activity location choice.

To attain a set of possible activity-travel plans of each synthetic individual, CEMDAP was used in this study. Multiple CEMDAP outputs, generated by varying the workplace and school locations in the input files, are created and fed into the MATSIM transport system simulation. The set of activity-travel plans of each synthetic traveler is considered a set of potential solutions to the problem of finding a valid transport demand representation. A calibration algorithm (CADYTS) is used to ensure that those initial suggestions of potential daily plans that contribute to reproducing real-world traffic patterns are selected. The procedure of feeding the output of an ABM into a dynamic traffic simulation in interaction with a calibration algorithm that manages the adequate selection of initial suggestions is novel and increases the transferability of transport demand models from one region (the estimation context) to another region (the application context).

The model created in this study validated very well. Lower mean relative errors for volumes of traffic are about 20\% during daytime hours ("with CADYTS" in Table 1 and Figure 2). The performance in regard to model fit is, thus, comparable with models based on travel diaries.

An independent validation, undertaken according to data from the Berlin $2008 \mathrm{SrV}$ travel survey, was successful concerning all considered properties (27). These properties encompass the total number of car trips and the distributions of departure times, trip duration, trip distance, and average trip speeds as well as the distribution of activity participation at trip ends.

To conclude, results suggest that it may be possible for a model estimated for one geographic region to be transferred to a different region. On the basis of publicly available input data of the new region and in interaction with a traffic-count-based updating of activity-travel plan scoring (CADYTS), an evolutionary simulation (MATSIM) may be able to generate a representative travel demand for the new region. Overall, the proposed approach appears quite encouraging in regard to developing policy-sensitive transport models for application contexts based on an estimated ABM in an estimation context combined with traffic count data from the application context.

\section{ACKNOWLEDGMENTS}

The authors thank Gunnar Flötteröd for help with CADYTS, Andreas Neumann for help with handling network and traffic counts, Subodh Dubey for technical assistance with the CEMDAP model, and the Berlin Senate Department for Urban Development and the Environment (Senatsverwaltung für Stadtentwicklung und Umwelt) for granting access to the Berlin SrV scientific use file. The last author acknowledges support from the Humboldt Foundation.

\section{REFERENCES}

1. Chiu, Y.-C., J. Bottom, M. Mahut, A. Paz, R. Balakrishna, T. Waller, and J. Hicks. Transportation Research Circular E-C153: Dynamic Traffic Assignment: A Primer. Transportation Research Board of the National Academies, Washington, D.C., 2011.

2. TRANSIMS. http://code.google.com/p/transims/. Accessed March 26, 2013.

3. DynusT. http://dynust.net. Accessed Aug. 12, 2013.

4. MATSIM. http://matsim.org. Accessed July 28, 2013. 
5. Bundesagentur für Arbeit. Pendlerstatistik 2010 (CD-ROM), 2010.

6. van Zuylen, H. J., and L. G. Willumsen. The Most Likely Trip Matrix Estimated from Traffic Counts. Transportation Research Part B, Vol. 14, 1980, pp. 281-293.

7. Flötteröd, G., M. Bierlaire, and K. Nagel. Bayesian Demand Calibration for Dynamic Traffic Simulations. Transportation Science, Vol. 45, 2011, pp. 541-561.

8. Flötteröd, G. Cadyts-Calibration of Dynamic Traffic SimulationsVersion 1.1.0 Manual. http://people.kth.se/ gunnarfl/files/cadyts/Cadyts manual_1-1-0.pdf. Accessed Nov. 12, 2014.

9. Flötteröd, G., Y. Chen, and K. Nagel. Behavioral Calibration and Analysis of a Large-Scale Travel Microsimulation. Networks and Spatial Economics, Vol. 12, 2011, pp. 481-502.

10. Moyo Oliveros, M., and K. Nagel. Automatic Calibration of AgentBased Public Transit Assignment Path Choice to Count Data. Presented at Conference on Agent-Based Modeling in Transportation Planning and Operations, Blacksburg, Va., 2013.

11. Bhat, C., J. Guo, S. Srinivasan, and A. Sivakumar. CEMDAP User's Manual. Center for Transportation Research, University of Texas, Austin, 2008.

12. Balmer, M., M. Rieser, A. Vogel, K. Axhausen, and K. Nagel. Generating Day Plans Using Hourly Origin-Destination Matrices. In Jahrbuch 2004/05 Schweizerische Verkehrswirtschaft (T. Bieger, C. Laesser, and R. Maggi, eds.), Schweizer Verkehrswissenschaftliche Gesellschaft, Saint Galler, Switzerland, 2005, pp. 5-36.

13. Rieser, M., K. Nagel, U. Beuck, M. Balmer, and J. Rümenapp. AgentOriented Coupling of Activity-Based Demand Generation with a Multiagent Traffic Simulation. In Transportation Research Record: Journal of the Transportation Research Board, No. 2021, Transportation Research Board of the National Academies, Washington, D.C., 2007, pp. 10-17.

14. Cambridge Systematics, Inc., Vanasse Hangen Brustlin, Inc., Gallop Corporation, C. R. Bhat, Shapiro Transportation Consulting, and Martin/ Alexiou/Bryson. NCHRP Report 716: Travel Demand Forecasting: Parameters and Techniques. Transportation Research Board of the National Academies, Washington, D.C., 2012.

15. Arentze, T., F. Hofman, H. van Mourik, and H. Timmermans. Spatial Transferability of the Albatross Model System: Empirical Evidence from Two Case Studies. In Transportation Research Record: Journal of the Transportation Research Board, No. 1805, Transportation Research Board of the National Academies, Washington, D.C., 2002, pp. 1-7.

16. Nowrouzian, R., and S. Srinivasan. Empirical Analysis of Spatial Transferability of Tour-Generation Models. In Transportation Research Record: Journal of the Transportation Research Board, No. 2302, Transportation Research Board of the National Academies, Washington, D.C., 2012, pp. 14-22.

17. Sikder, S., and A.R. Pinjari. Spatial Transferability of Person-Level Daily Activity Generation and Time-Use Models: Empirical Assessment. In Transportation Research Record: Journal of the Transportation
Research Board, No. 2343, Transportation Research Board of the National Academies, Washington, D.C., 2013, pp. 95-104.

18. Bowman, J. L., M. Bradley, J. Castiglione, and S. L. Yoder. Making Advanced Travel Forecasting Models Affordable Through Model Transferability. Presented at 93rd Annual Meeting of the Transportation Research Board, Washington, D.C., 2014.

19. Bhat, C. R., J. Y. Guo, S. Srinivasan, and A. Sivakumar. A Comprehensive Econometric Microsimulator for Daily Activity-Travel Patterns. In Transportation Research Record: Journal of the Transportation Research Board, No. 1894, Transportation Research Board of the National Academies, Washington, D.C., 2004, pp. 57-66.

20. Pendyala, R., C. Bhat, K. Goulias, R. Paleti, K. Konduri, R. Sidharthan, and K. Christian. SimAGENT Population Synthesis. GeoTrans Laboratory, University of California, Santa Barbara, 2013.

21. Bhat, C., K. Goulias, R. Pendyala, R. Paleti, R. Sidharthan, L. Schmitt, and H.-H. Hu. A Household-Level Activity Pattern Generation Model with an Application for Southern California. Transportation, Vol. 40, 2013, pp. 1063-1086.

22. Gawron, C. Simulation-Based Traffic Assignment. PhD dissertation. University of Cologne, Germany, 1998.

23. Charypar, D., and K. Nagel. Generating Complete All-Day Activity Plans with Genetic Algorithms. Transportation, Vol. 32, 2005, pp. 369-397.

24. Neumann, A., M. Balmer, and M. Rieser. Converting a Static Trip-Based Model into a Dynamic Activity-Based Model to Analyze Public Transport Demand in Berlin. In Travel Behaviour Research: Current Foundations, Future Prospects (M. Roorda and E. Miller, eds.), International Association for Travel Behaviour Research, 2014, pp. 151-176.

25. Amt für Statistik Berlin-Brandenburg. Bevölkerungsstand in Berlin am 31. Dezember 2012 nach Bezirken. https://www.statistik-berlin -brandenburg.de/Publikationen/OTab/2013/OT_A01-10-00_124 _201212_BE.pdf. Accessed Nov. 4, 2013.

26. Bömermann, H., S. Jahn, and K. Nelius. Lebensweltlich orientierte Räume im Regionalen Bezugssystem (Teil 1). Berliner Statistik, Vol. 8, 2006, pp. 366-371.

27. Ziemke, D. Demand Generation for Multi-Agent Transport Simulations Based on an Econometric Travel Behavior Model and a Traffic-CountBased Calibration Algorithm. Master's thesis. Technische Universität Berlin, 2013.

28. Ahrens, G.-A. Endbericht zur Verkehrserhebung Mobilität in StädtenSrV 2008 in Berlin. Institut für Verkehrs-und Infrastrukturplanung, Dresden University of Technology, Germany, 2009.

29. Flötteröd, G., Y. Chen, and K. Nagel. Choice Model Refinement from Network Data. Proc., 13th Conference of the International Association for Travel Behavior Research, Toronto, Canada, 2012.

The Standing Committee on Transportation Demand Forecasting peer-reviewed this paper. 